\title{
Acute mesenteric lymphadenitis in children: findings related to differential diagnosis and hospitalization
}

\author{
Mustafa Yaşar Özdamar ${ }^{1}$ Erdal Karavaş
}

\author{
${ }^{1}$ Department of Pediatric Surgery, Faculty of Medicine, Erzincan Binali Yildirim \\ University, Erzincan, Turkey \\ 2Department of Radiology, Faculty of Medicine, Erzincan Binali Yildirim University, \\ Erzincan, Turkey
}

Submitted: 14 August 2018

Accepted: 20 September 2018

Arch Med Sci 2020; 16 (2): 313-320

DOI: https://doi.org/10.5114/aoms.2018.79430

Copyright $\odot 2018$ Termedia \& Banach

\section{Abstract}

Introduction: Diagnostic discrimination between acute appendicitis (AA) and mesenteric lymphadenitis (AML) may require more diagnostic tests or great skill after excluding other diagnoses. This study aimed to make a differential diagnosis between AA and AML patients with previous and new parameters and to examine which parameters should be taken into account regarding whether the AML patient should be hospitalized or discharged.

Material and methods: One hundred and twenty-three AML and $134 \mathrm{AA}$ patients, randomly selected, were included in the study. Demographic, clinical, and laboratory data of all subjects were analyzed. Ultrasonographic and rarely computed tomography examinations evaluating for the enlarged lymph nodes with the shortest diameter in the right lower quadrant of the AML patients were performed. Also, the erect abdominal radiographs (EAR) of $A M L$ and $A A$ patients were evaluated.

Results: While there was no statistically significant difference in age or gender, C-reactive protein, white blood cell count, monocyte percentage as well as symptoms between the AA and AML groups $(p>0.05)$, neutrophil and lymphocyte percentage, appearance of EAR and L/M ratio were significantly different between the two groups $(p<0.05)$. There was no correlation between the short-axis diameter of the mesenteric lymph node and clinical and laboratory findings in the AML group $(p>0.05)$.

Conclusions: Based on the results with ROC curve analysis, we propose to take into account the L/M ratio and the EAR from the parameters in the differential diagnosis between $A A$ and $A M L$ in addition to previously reported parameters. Also, we recommend that the same features may be used to decide whether AML patients under observation are hospitalized or not.

Key words: mesenteric lymphadenitis, acute appendicitis, children, differential diagnosis.

\section{Introduction}

The most common surgical disease in children with acute abdominal pain admitted to the emergency department is acute appendicitis (AA). Diagnostic discrimination between AA and acute mesenteric lymphadenitis (AML) may sometimes be difficult as a result of the physical examination and laboratory studies after excluding other diagnoses such as diarrhea, urinary infection, intussusception, and gastroenteritis [1, 2]. Acute mesenteric lymphadenitis causes right lower quadrant (RLQ) pain

\author{
Corresponding author: \\ Mustafa Yaşar Özdamar \\ MD, Asst. Prof. \\ Department of \\ Pediatric Surgery \\ Faculty of Medicine \\ Erzincan Binali \\ Yildirim University \\ 24000 Erzincan, Turkey \\ Phone: +90 4462122215 \\ +905062418001 \\ Fax: +90 446.212 2218 \\ E-mail: \\ mustafayasarozdamar@ \\ gmail.com
}


as in AA and its etiology may be due to primary (idiopathic) or secondary (infection, malignancy, etc.) reasons [3].

Although AML brings about abdominal pain, it does not cause any abnormalities in many healthy children with enlarged mesenteric lymph nodes. Wang et al. asserted in an ultrasound-guided clinical study that the mesenteric lymph nodes increase with age until 6 years and then decrease. Moreover, they supposed that AML with a short axis diameter larger than $8 \mathrm{~mm}$ could be related to abdominal pain [4]. Also, in another study, the short axis diameter of larger than $8 \mathrm{~mm}$ and even $10 \mathrm{~mm}$ was suggested for the definition of the pathologic mesenteric lymph node in children [5].

The consensus regarding the radiological and laboratory parameters which may be consistent with $A M L$ in the literature is still controversial $[6,7]$. The most common gastrointestinal complaints in AML are abdominal pain, nausea-vomiting, fever, and loose stools, respectively. The clinical and laboratory results of AA and AML may create uncertainty about what differential diagnosis will be made for most practitioners. While establishing a relationship between the clinic picture and mesenteric lymphadenitis (ML), patients with the short axis diameter of ML greater than $10 \mathrm{~mm}$ were excluded from the study in some studies; the others accepted that $M L$ with a short-axis diameter of $8 \mathrm{~mm}$ or more constituted AML $[5,8,9]$. Erect abdominal radiographs (EAR) can provide supportive findings in the differential diagnosis of intestinal obstruction or ileus in children [10]. However, no study has made a differential diagnosis by establishing a relationship between EAR and the AML or AA patients' clinical characteristics.

This study aimed to evaluate the clinical and laboratory parameters of patients with symptomatic $A M L$ and to compare them with those of $A A$ patients, and also to further investigate those parameters based on lymph node size. Also, we examined which parameters should be taken into account regarding whether the AML patient should be hospitalized or discharged.

\section{Material and methods}

Data of all patients aged 5-16 years who consecutively presented in the Emergency Department and the outpatient clinic of Pediatric Surgery between the dates July 2015 and May 2018 and were diagnosed with $A M L$ and $A A$ were retrospectively analyzed. The study was approved by the Institutional Ethics Review Board for Clinical Research of Erzincan Binali Yildirim University (2018, 24/5). Patients with missing data were removed from the study. Demographic, clinical, and laboratory data of all subjects were analyzed. The abdominal ultrasound (US) was performed by a ra- diologist using the HI VISION Preirus scanner with a curved-array $(2-5 \mathrm{MHz})$ and a linear-array transducer (6-13 MHz; Hitachi Medical Corporation, Japan). Ultrasonographic and rarely computed tomography (CT) examinations evaluating for the enlarged lymph nodes with the shortest diameter in the RLQ of the AML patients were performed. Also, the erect abdominal radiographs of $A M L$ and AA patients were evaluated. The US finding of AA was the aperistaltic, incompressible, dilated appendix with $>6 \mathrm{~mm}$ diameter. AA was precisely identified according to pathological specimen results after surgery. Symptomatic AML and AA patients were detected according to the discharge summary in which there was found right lower quadrant (RLQ) pain, rebound or tenderness findings of the patients.

\section{Statistical analysis}

We performed statistical analysis to for evaluate our results using SPSS Statistics for Windows version 18.0 software (SPSS Inc., USA). When the sample size was less than 50, the Shapiro-Wilk test was used for normality of distribution concerning the data evaluation. Otherwise, the Kolmogorov-Smirnov test was used. For two independent groups, nonparametric tests, such as the Mann Whitney- $U$ test, were used in the case of rejection; otherwise, a $t$-test was used. The Kruskal-Wallis test was used when the number of groups for the nonparametric data was low. Correlation between categorical variables was analyzed by the $\chi^{2}$ test. Correlation between numeric variables was examined by the Pearson test. We used Levene's test to assess the equality of variances and a two-sided 95\% confidence interval for all measures. Statistical significance was defined at $p<0.05$. For the statistically significant result between the groups in the laboratory test used in differential diagnosis, the sensitivity, specificity, and cut-off value were calculated using the receiver operating characteristic (ROC) curve. The area under the curve of 0.50 represented a variable with no differential capacity, and the area of 1.00 indicated a perfect discriminator.

\section{Results}

A total of 2680 (100\%) patients with abdominal pain were determined. The AA in 134 (5\%), AML in 123 (4.5\%), constipation in 450 (16.7\%), diarrhea in $650(24.2 \%)$, and urinary tract infection in 442 (16.5\%) patients were reasons for abdominal pain. Also, incarcerated inguinal hernia, ovarian torsion, testicular torsion, invagination, and diabetic ketoacidosis, etc. in 77 (3.1\%) cases, and upper/lower respiratory tract infection in 804 (30\%) patients were other reasons for abdominal pain. 
Table I. Comparison of age and gender between AA and AML groups and within each group

\begin{tabular}{|c|c|c|c|c|}
\hline Parameter & $N(\%)$ & Age, mean \pm SD & $P$-value (age) & $P$-value (gender) \\
\hline AA: & & & 0.72 & 0.65 \\
\hline Male & $64(52)$ & $9.71 \pm 2.54$ & & \\
\hline Female & $59(48)$ & $9.87 \pm 2.22$ & & \\
\hline Total & $123(100)$ & $9.76 \pm 2.43$ & & \\
\hline AML: & & & 0.50 & 0.73 \\
\hline Male & $69(52)$ & $9.80 \pm 2.81$ & & \\
\hline Female & $65(48)$ & $9.48 \pm 2.08$ & & \\
\hline Total & $134(100)$ & $9.70 \pm 2.58$ & & \\
\hline AA vs. AML & $257(100)$ & $9.73 \pm 2.51$ & 0.84 & 0.86 \\
\hline
\end{tabular}

$A A$ - group of acute appendicitis, $A M L$ - group of acute mesenteric lymphadenitis.

One hundred and twenty-three AML patients and 134 AA patients without loss of data were consecutively detected. In 24 (9.7\%) out of 247 patients with intensive intestinal gas or obesity, CT was used instead of USG because the diagnosis and differential diagnosis could not be reliably performed. The final diagnosis of AML or AA was made with the US and CT examination. Also, AA diagnosis was confirmed by the patient's pathology and operative report, and the postoperative diagnosis of all preoperative AA patients was the same. There was no significant difference in age or gender between the two groups or within each group ( $p>0.05$; Table I).

RLQ tenderness, abdominal guarding, and rebound tenderness, which were the findings of RLQ pain in patients, were present in the examination of all patients excluding five AA and nine AML patients who were admitted for only vomiting and
$\mathrm{AA}$ and $\mathrm{AML}$ were detected on ultrasound examination, respectively (Table II).

None of the AML patients were detected to have wall thickening of any bowel segment. According to retrospective data in both groups, symptoms were divided into three variables: 1 , only RLQ pain; 2, RLQ pain and vomiting; 3, only vomiting. From the complete blood count results, white blood cells (WBC; reference $\left.<12.5 \times 10^{3} / \mu \mathrm{l}\right)$, neutrophil $(\mathrm{N})$, lymphocyte $(\mathrm{L})$ and monocyte $(\mathrm{M})$ percentage, as well as $L / M$ ratio were obtained. C-reactive protein (CRP; reference < $10 \mathrm{mg} / \mathrm{dl}$ ) records of all patients were collected. Erect $a b$ dominal radiographs (EAR) were taken from the radiological records, and they were separated into three groups: A - gas-fluid level present; B - the gas-fluid level is absent, but abundant gas exists; $\mathrm{C}$ - normal appearance. Patients who did not meet these parameters were not included in the study.

Table II. Comparison of AA and AML groups' data including erect abdominal radiography, symptoms and complete blood count results

\begin{tabular}{|lccc|}
\hline Parameter & AA $(n=123)$ & AML $(n=134)$ & $P$-value \\
\hline CRP $($ mean \pm SD) $[\mathrm{mg} / \mathrm{dl}]$ & $10.56 \pm 4.21$ & $10.21 \pm 4.56$ & 0.53 \\
\hline WBC $\left(\right.$ mean \pm SD) $\left[\times 10^{3} / \mu \mathrm{l}\right]$ & $10.80 \pm 2.66$ & $10.28 \pm 2.23$ & 0.18 \\
\hline Neutrophils $(\mathrm{N}) \%$ & $76.13 \pm 3.66$ & $58.15 \pm 10.89$ & 0.00 \\
\hline Lymphocytes (L) \% & $10.76 \pm 3.66$ & $32.27 \pm 11.58$ & 0.00 \\
\hline Monocytes (M) \% & $5.70 \pm 1.80$ & $6.64 \pm 5.07$ & 0.15 \\
\hline L/M ratio & $2.06 \pm 0.86$ & $5.52 \pm 1.63$ & 0.00 \\
\hline Symptoms, $n(\%):$ & $123(100)$ & $134(100)$ & 0.66 \\
\hline 1 & $69(56)$ & $63(47)$ & \\
\hline 2 & $49(40)$ & $62(46)$ & \\
\hline 3 & $5(4)$ & $9(7)$ & \\
\hline EAR, $n(\%):$ & $123(100)$ & $134(100)$ & \\
\hline A & $76(62)$ & $52(39)$ & \\
\hline B & $42(34)$ & $31(23)$ & \\
\hline C & $5(4)$ & $513)$ & \\
\hline
\end{tabular}

$A A$ - group of acute appendicitis, $A M L$ - group of acute mesenteric lymphadenitis. Symptoms: 1 - only RLQ pain, $2-R L Q$ pain and vomiting, 3 - only vomiting. EAR - Erect abdominal radiograph: $A$ - gas-fluid level present, B - gas-fluid level is absent but abundant gas exists, $C$ - normal appearance. 
As shown in Table II, there was no statistically significant difference between the two groups except for $\mathrm{N}$ and $\mathrm{L}$ percentage, $\mathrm{EAR}$, and $\mathrm{L} / \mathrm{M}$ ratio during the first evaluation (Table II, $p<0.05$ ). When the ROC curve for parameters in the discrimination of groups was plotted, the parameters with the largest area under the curve (AUC) were $\mathrm{N}$ and $\mathrm{L}$ percentage, L/M ratio, and EAR (Figure 1). Cut-off value, sensitivity and specificity for $\mathrm{N}, \mathrm{L}$ percentage and L/M ratio were $80.9,10 \%$ and $89 \%$; 48.7 , $20 \%$ and $94 \%$; as well as $8.95,89 \%$ and $97 \%$, respectively.

Observation status was conferred for AML patients considered not ill enough to be fully admitted but not well enough to go home especially due to vomiting and pain. To decide which patient

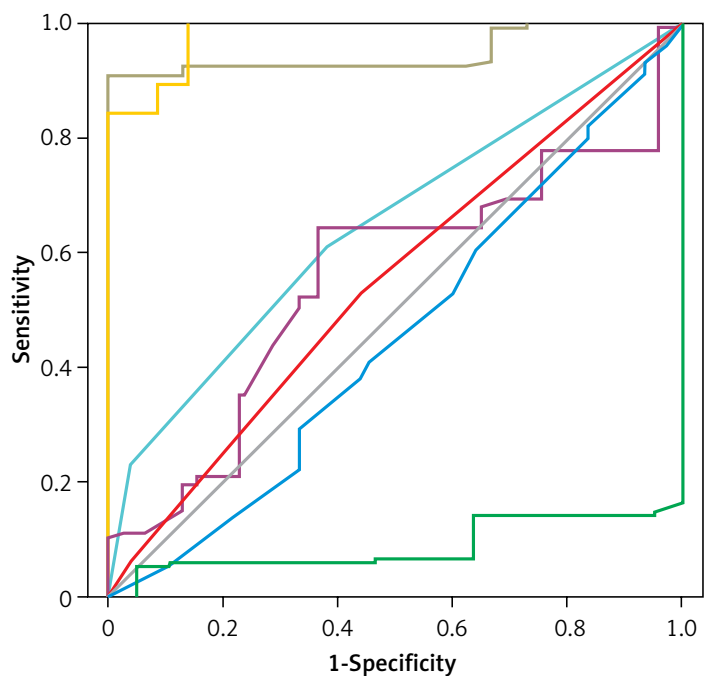

should be admitted to the hospital the same parameters as above were used. AML patients under observation were reevaluated after about $8 \mathrm{~h}$ (range: 4-8 h) of symptomatic treatment was applied especially for pain and nausea-vomiting. US findings of the hospitalized AML patients were usually a peristaltic, compressible appendix with 6-8 $\mathrm{mm}$ diameter. As the cause of large diameter of the appendix together with some AML patients could be a doubtful case of appendicitis, other findings were evaluated. We reevaluated the new EAR and current symptoms of them. As shown in Table III, there was no statistically significant difference between the hospitalized and discharged AML patients except for EAR (Figure 2), symptoms and $\mathrm{L} / \mathrm{M}$ ratio $(p<0.05)$. When the ROC curve for

\begin{tabular}{|l|c|c|}
\hline Variables & Area under the curve & $P$-value \\
\hline WBC & 0.452 & 0.182 \\
\hline N & 0.088 & $<0.001$ \\
\hline L & 0.948 & $<0.001$ \\
\hline M & 0.551 & 0.155 \\
\hline L/M & 0.981 & $<0.001$ \\
\hline Symptom & 0.549 & 0.171 \\
\hline EAG & 0.647 & $<0.001$ \\
\hline
\end{tabular}

Source of the curve:

$-\mathrm{WBC} \quad \mathrm{N} \quad \mathrm{L} \quad \mathrm{M} \quad \mathrm{L} / \mathrm{M} \quad$ Symptom $\quad \mathrm{EAG} \quad$ Reference line

Figure 1. ROC curve for parameters in the discrimination of groups (AA and $A M L)$

Table III. Comparison of hospitalization and discharge characteristics in AML patients under observation

\begin{tabular}{|c|c|c|c|}
\hline Parameter & Hospitalized AML* & Discharged AML & $P$-value \\
\hline WBC $\left(\right.$ mean \pm SD) $\left[\times 10^{3} / \mu \mathrm{l}\right]$ & $10.42 \pm 1.51$ & $10.26 \pm 2.33$ & 0.29 \\
\hline Neutrophils (N) (\%) & $58.21 \pm 14.23$ & $58.09 \pm 10.28$ & 0.78 \\
\hline Lymphocytes (L) (\%) & $38.38 \pm 7.23$ & $11.13 \pm 1.04$ & 0.62 \\
\hline Monocytes (M) (\%) & $7.39 \pm 2.37$ & $7.39 \pm 1.95$ & 0.46 \\
\hline $\mathrm{L} / \mathrm{M}$ ratio & $6.48 \pm 1.90$ & $5.09 \pm 1.55$ & 0.02 \\
\hline Symptoms, $n(\%)$ : & $20(100)$ & $114(100)$ & 0.00 \\
\hline 1 & $0(0)$ & $99(87)$ & \\
\hline 2 & $12(60)$ & $0(0)$ & \\
\hline 3 & $8(40)$ & $15(13)$ & \\
\hline $\mathrm{EAR}, n(\%):$ & $20(100)$ & $114(100)$ & 0.004 \\
\hline$A$ & $18(90)$ & $34(30)$ & \\
\hline $\mathrm{B}$ & $2(10)$ & $49(43)$ & \\
\hline $\mathrm{C}$ & $0(0)$ & $31(27)$ & \\
\hline
\end{tabular}

$A A$ - group of acute appendicitis, $A M L$ - group of acute mesenteric lymphadenitis. Symptoms: 1 - Only RLQ pain, $2-R L Q$ pain and vomiting, 3 - only vomiting. EAR - erect abdominal radiograph; $A$-gas-fluid level present, $B$-gas-fluid level is absent but abundant gas exists, $C$-normal. *AML patients not fully recovering from nausea-vomiting and pain after 8 hours of symptomatic treatment. 

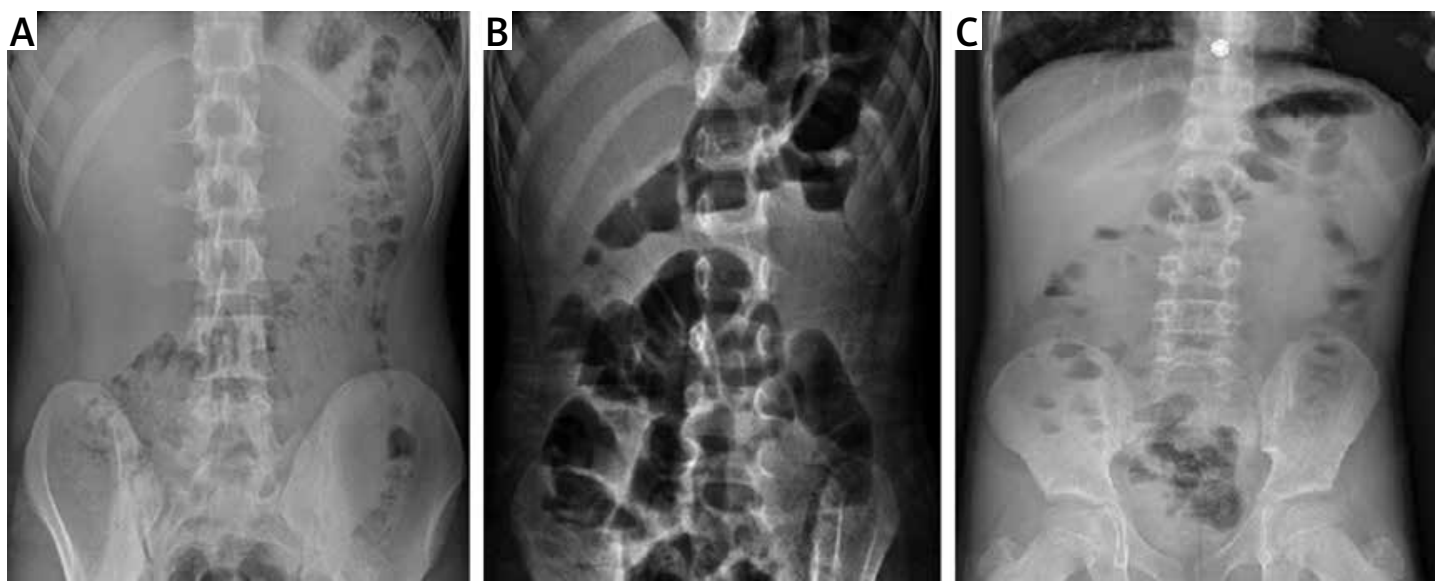

Figure 2. Abdominal erect radiographs of three distinct AML patients. A - Normal X-ray of a discharged patient. B - Gas-fluid levels present in the X-ray of a hospitalized patient. C - A hospitalized patient with small gas pockets and small gas-fluid levels (A, B and C patients with a normal appendix on the US examination)

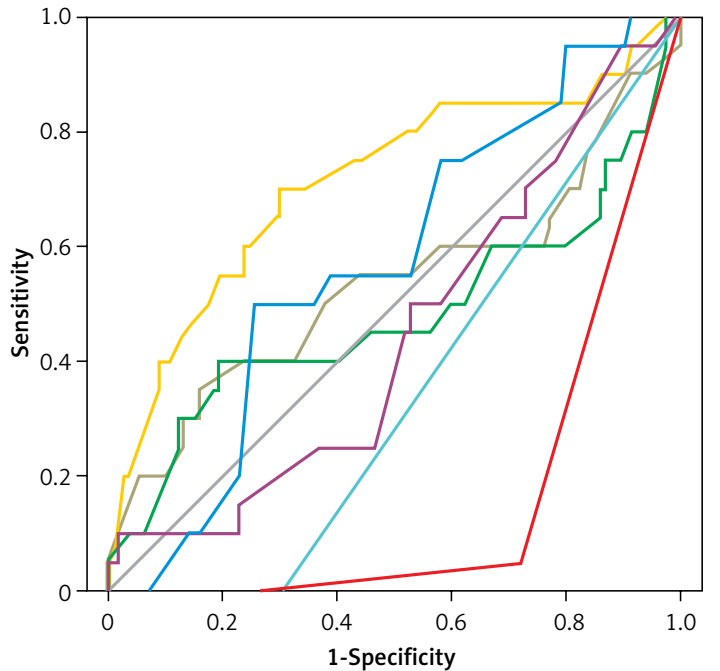

\begin{tabular}{|l|c|c|}
\hline Variables & Area under the curve & $P$-value \\
\hline WBC & 0.574 & 0.294 \\
\hline N & 0.481 & 0.788 \\
\hline L & 0.534 & 0.626 \\
\hline M & 0.448 & 0.461 \\
\hline L/M & 0.715 & 0.002 \\
\hline EAR & 0.159 & $<0.001$ \\
\hline Symptom & 0.346 & 0.029 \\
\hline
\end{tabular}

- WBC

Source of the curve:

igure 3. ROC curves for indicating whether patients with AML should be hospitalized or discharged

parameters in the discrimination of groups was plotted, the parameter with the largest area under the curve was only L/M ratio (Figure 3 ). Cut-off value, sensitivity and specificity for $\mathrm{L} / \mathrm{M}$ ratio were $8.95,89 \%$ and $97 \%$, respectively.

On the ultrasound examination, we detected a cluster of three or more enlarged lymph nodes in the RLQ and mesentery of the ileocaecal region or the anterior to the psoas, and also measured the short-axis diameter of the largest lymph node in all patients with AML. We found that there was no significant correlation between all parameters of AML patients and the short axis diameter of the largest lymph node $(p>0.05)$; Table IV). As shown in Figure 4 and Table III, the number of patients with a short axis diameter of the largest lymph node less than $8 \mathrm{~mm}$ was 118 (88\%). These results indicated that the parameters constituting the clinical condition were not affected by the lymph node size.

\section{Discussion}

When looking at the literature, there was generally no significant difference in age (range: 9-13) or gender in studies comparing AML and AA patients $[8,11]$. Conversely, there was a study that found significant differences in age between $A A$ and AML patients (male vs. female: 10.3 vs. 7.9 years) [7]. This difference may be due to the fact that the regions where these studies were carried out are different (Israel, Latvia and India). In our study, there was no statistically significant difference in age (mean: $9.73 \pm 2.51$ years) or gender between the AA and AML groups. Also, there was no significant difference regarding age or gender among the patients of the AML group. The same characterization was also present in the AA group (Table I, $p>0.05$ ).

The AML has a clinical presentation that can mimic $A A$, and this dilemma may lead to a nega- 
Table IV. Correlation of short-axis diameter of mesenteric lymph node with clinical and laboratory findings in the AML group

A

B

\begin{tabular}{|c|c|c|c|c|c|}
\hline \multicolumn{2}{|l|}{$\begin{array}{l}\text { AML group } \\
\text { Parameters }\end{array}$} & \multirow{2}{*}{$\begin{array}{c}\text { A\&B correlation } \\
P \text {-value }\end{array}$} & \multicolumn{3}{|c|}{$\begin{array}{l}\text { Short axis diameter of the largest lymph node } \\
\qquad[\mathrm{mm}]\end{array}$} \\
\hline WBC $($ mean $\pm S D)\left[\times 10^{3} / \mu \mathrm{l}\right]$ & $10.28 \pm 2.23$ & & \multirow{2}{*}{\multicolumn{3}{|c|}{$\begin{array}{l}(\text { mean } \pm S D ; 5.62 \pm 2.26) \\
(\text { rank: } 3-12 \mathrm{~mm})\end{array}$}} \\
\hline $\mathrm{CRP}($ mean $\pm \mathrm{SD})[\mathrm{mg} / \mathrm{dl}]$ & $10.21 \pm 4.56$ & 0.16 & & & \\
\hline Lymphocytes (L) (\%) & $32.27 \pm 11.58$ & 0.42 & Diameter & $N$ & Percent \\
\hline Monocytes (M) (\%) & $6.64 \pm 5.07$ & 0.40 & 3.00 & 18 & 13.4 \\
\hline $\mathrm{L} / \mathrm{M}$ ratio & $5.52 \pm 1.63$ & 0.90 & 4.00 & 30 & 22.4 \\
\hline Symptoms, $n$ (\%): & $134(100)$ & 0.44 & 5.00 & 39 & 29.1 \\
\hline 1 & $63(47)$ & & 6.00 & 12 & 9.0 \\
\hline 2 & $62(46)$ & & 7.00 & 6 & 4.5 \\
\hline 3 & $9(7)$ & & 8.00 & 13 & 9.7 \\
\hline EAR, $n(\%):$ & $134(100)$ & 0.48 & 9.00 & 3 & 2.2 \\
\hline A & $52(39)$ & & 10.00 & 7 & 5.2 \\
\hline $\mathrm{B}$ & $51(38)$ & & 11.00 & 3 & 2.2 \\
\hline \multirow[t]{2}{*}{ C } & \multirow[t]{2}{*}{$31(23)$} & & 12.00 & 3 & 2.2 \\
\hline & & & Total & 134 & 100.0 \\
\hline
\end{tabular}

$A M L$ - acute mesenteric lymphadenitis. Symptoms: 1 - Only RLQ pain, $2-R L Q$ pain and vomiting, 3-only vomiting. EAR - erect abdominal radiograph: $A$-gas-fluid level present, $B$-gas-fluid level is absent but abundant gas exists, $C$-normal appearance.

tive exploration in the operations performed with AA pre-diagnosis [6, 12]. Various clinical and laboratory parameters have been used in studies to cope with this difficulty. $\mathrm{N}$ to $\mathrm{L}$ count ratio in the diagnosis of AA was established and this parameter was accepted as a positive predictor in favor of AA. Moreover, Gross et al. suggested that lymphocytosis is a positive predictor in favor of $A M L$ regarding the differential diagnosis between $A M L$ and AA $[6,13]$. Similarly, in our study, $N$ percentage was higher in $A A$ and $L$ percentage was higher in AML patients. Differently, in our study, the L/M ratio was significantly higher in the $A M L$ group

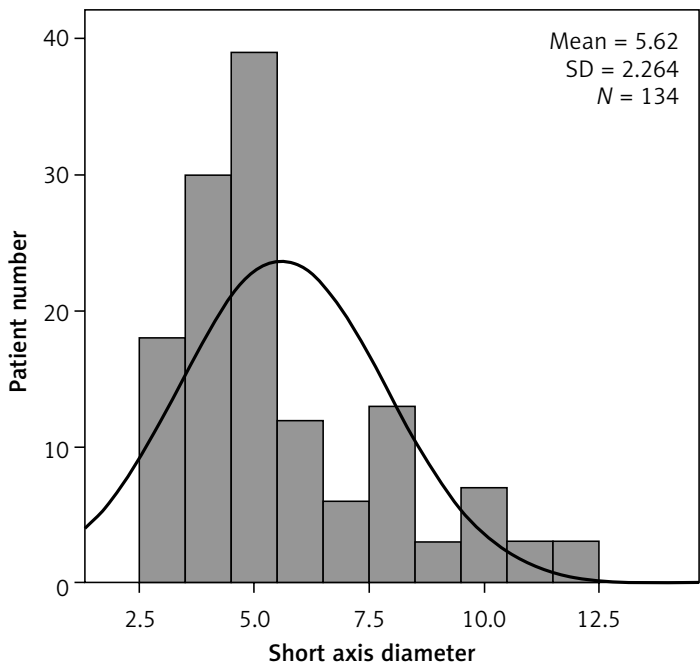

Figure 4. Histogram showing distribution of a short axis diameter measurement of the largest lymph node according to AML patient numbers than that of the AA group (Table II, $p<0.001$ ). The percentage of $M$ alone was not different between the two groups. In a study performed for AA and AML differential diagnosis, the monocyte chemoattractant protein-1 (MCP 1) ratio was found not to be different between these two groups. In this study, although the MCP 1 ratio was high in AA, there was no statistically significant difference between the two groups [11]. This result was compatible with our work regarding the $M$ percentage in the discrimination of both groups $(p=0.15)$. The CRP and WBC rates were higher in favor of $A A$ in a recent study and this result was statistically significant [6]. There was, however, no significant difference in WBC and CRP between the two groups in our study $(p>0.05)$. As shown in Table II, when $\mathrm{L}$ and $\mathrm{N}$ are within normal limits, the $\mathrm{L} / \mathrm{M}$ ratio seems to be more reliable when making a differential diagnosis between $A A$ and $A M L$. In a recent study, higher values of $N / L$ and $M / L$ ratio demonstrated greater possibilities for bacterial infection and low probabilities for viral infection [14]. Rothrock et al. found that initial misdiagnosis in childhood appendicitis included gastroenteritis and upper respiratory tract infection in the percentage of $60 \%[8,15] . M / L$ and $N / L$ ratio can help the practitioner to differentiate these diseases since $A M L$ has the same confusion and condition in the differential diagnosis of $\mathrm{AA}$, and because the viral agents are often considered in the etiology of these involved diseases regarding discrimination of AA. When considering the above pieces of evidence, we tried to make a differen- 
tial diagnosis between $A A$ and $A M L$, taking into account the $L / M$ ratio instead of the $M / L$ ratio, and to facilitate the diagnosis of $A M L$, which is more likely to be viral by etiology.

Even though the Alvarado score and the pediatric appendicitis score described by Samuel were efficiently used for the diagnosis of AA [7, 16, 17], some authors in their prospective studies reported that both scores could be useful parameters for the diagnosis of AA; however, they concluded that these scores were insufficient to determine whether surgical intervention is required or not [18-20]. In other words, it has been revealed that differential diagnosis cannot be made with current symptoms. According to the parameters we collected from the archive records, abdominal pain percentage was higher in the AA group, while nausea-vomiting was higher in the $A M L$ group, but these were statistically insignificant in the differential diagnosis $(p=0.66)$. In this context, complementary radiological examinations were needed for differential diagnosis and correct treatment. Because of this, CT examination was recommended in cases where the differential diagnosis between $A A$ and $A M L$ was not possible with the US examination [7].

We traditionally used EARs [21] in patients in whom we could not make a differential diagnosis with US. Thus, with the CT examination, patients were not exposed to more radiation in the first assessment. When the appendix could not be visualized due to abundant intestinal gas on the US examination, or it could not be determined in any area of the RLQ, we first looked at the patient's L/M ratio. We observed the patients with an $L / M$ ratio of 5 and above. We then used the EAR to assess intestinal stability. We used CT examination (about 9.7\%, 24 out of 257 patients) in patients with an L/M ratio less than 5 . Although there was no diarrhea in the AML patients under observation (in all of the AML patients hospitalized and in some of the subjects discharged) their EARs had gas-liquid levels or small gas pockets (Figures $2 \mathrm{~B}, \mathrm{C}$ ). AML patients not fully recovering from nausea-vomiting and abdominal pain after $8 \mathrm{~h}$ of symptomatic treatment were hospitalized by looking at their new EARs and L/M ratio. While vomiting was more frequent in the hospitalized AML patients, abdominal pain was more frequent in discharged patients, and this clinical difference was statistically significant $(p<0.001)$. Moreover, while $90 \%$ of the hospitalized AML patients had EARs with gas-fluid levels, $70 \%$ of the discharged AML patients had EARs with normal gas distribution and small air pockets, which was statistically significant as well ( $p=0.004)$.

As shown in Table II, there was no significant correlation between the short-axis diameter of the mesenteric lymph node and clinical or laboratory findings in the AML group ( $p>0.05)$. However, it has been stated in the literature that especially abdominal pain occurred in AML patients with the short-axis diameter of the mesenteric lymph node over 8 or $10 \mathrm{~mm}$. Also, it has been supposed that a cluster of three or more lymph nodes measuring more than $5 \mathrm{~mm}$ along their short axis in AML was the pathologic condition [3, 4, 7, 22]. In our study, we found that abdominal pain and nausea-vomiting occurred at different rates independently of the short-axis diameter of the mesenteric lymph node (Figure 4, Table IV). In a recent study, there were no significant differences between laboratory and clinical parameters and lymph node size [6]. We have also shown that there is no correlation between EAR results and lymph node size.

In the ROC curve analysis, the L/M ratio was the most specific and the most sensitive parameter we used when making a differential diagnosis between AA and AML groups (Figure 1). We found that the previously reported percentage of $L$ [6] was less sensitive and specific. We detected that the same result presented in the ROC curves for indicating whether patients with AML could need to be hospitalized or discharged (Figure 2).

Since our study was retrospective, we could not obtain the body temperature precisely from the records. However, it was previously reported that high fever did not show any significant difference in the differential diagnosis [7]. Unlike adults, discriminating between children with and without appendicitis usually requires more effort. In other words, a $100 \%$ reliable laboratory test and the clinical feature have not yet been reported in the differential diagnosis. There is a need for more specific studies regarding the inflammatory process for $A A$ and $A M L$. For example, cytokines such as IL-1 $\beta$, IL-1ra, and IL-6 used for the clinical approach to ulcerative colitis relapse can be investigated in AA and $A M L[12,23,24]$. Colonoscopy may be used for higher diagnostic reliability of AML in children as in a recent study in which esophagogastroduodenoscopy was performed in children with chronic abdominal pain [25]. Thus, in an acute stage of $A M L$, colonoscopy may detect the intestinal changes which could be associated with AML by the same logical approach. In differential diagnosis, anamnestic factors are also important; for example, when the abdominal pain of a patient with chronic renal failure is questioned, it is malpractice to try to make the $A A$ or $A M L$ discrimination instead of performing dialysis-related tests immediately [26].

Another limitation is that, as in Gross's study [6], the analysis of results based on three categories may not be as realistic as a prospective study for the parameter under the heading "symptoms" associated with the physical examination. Although the EAR is used less frequently in clinical practice, it is still used, for example, in the diag- 
nosis of intestinal perforation [27]. In addition to the approaches mentioned above, we made a differential diagnosis of diseases such as intestinal perforation or intussusception via EAR.

In conclusion, when a child with abdominal pain is admitted to the emergency department, a complete blood count should be performed after the physical examination. Even if the WBC is within standard limits, the L/M ratio seems to be reliable for initial differential diagnosis between $A A$ and $A M L$. If it is not found that there is no appendicitis sign in the US, after this stage, it would be diagnosed in some diseases other than AA and AML using EAR, and thus patients will not be exposed to more radiation with CT. Computed tomography examination, which is one of the best technological facilities, is recommended in cases where the differential diagnosis between AA and AML is not possible with the USG examination or EAR made after USG examination.

Based on these results, we propose to take into account the $L / M$ ratio and EAR from the parameters in the differential diagnosis between AA and AML in addition to previously reported parameters. Also, we recommend that the same features be used to determine whether AML patients under observation are hospitalized or not. But there is a need for prospective studies on the accuracy and effectiveness of all the parameters we used.

\section{Conflict of interest}

The authors declare no conflict of interest.

\section{References}

1. Puylaert JB, van der Zant FM. Mesenteric lymphadenitis or appendicitis? AJR Am J Roentgenol 1995; 165: 490.

2. Kim JS. Acute abdominal pain in children. Pediatr Gastroenterol Hepatol Nutr 2013; 16: 219-24.

3. Macari M, Hines J, Balthazar E, Megibow A. Mesenteric adenitis: CT diagnosis of primary versus secondary causes, incidence, and clinical significance in pediatric and adult patients. Am J Roentgenol 2002; 178: 853-8.

4. Wang WG, Tian H, Yan JY, et al. Enlarged mesenteric lymph nodes in children: a clinical analysis with ultrasonography and the implications. Nan Fang Yi Ke Da Xue Xue Bao 2011; 31: 522-4.

5. Simanovsky N, Hiller N. Importance of sonographic detection of enlarged abdominal lymph nodes in children. J Ultrasound Med 2007; 26: 581-4.

6. Gross I, Siedner-Weintraub Y, Stibbe S, et al. Characteristics of mesenteric lymphadenitis in comparison with those of acute appendicitis in children. Eur J Pediatr 2017; 176: 199-205

7. Toorenvliet B, Vellekoop A, Bakker R, et al. Clinical differentiation between acute appendicitis and acute mesenteric lymphadenitis in children. Eur J Pediatr Surg 2011; 21: 120-3.

8. Maheswari K, Wadhwa L. Clinical profile and causes of mesenteric lymphadenitis in children. Int J Contemp Pediatr 2016; 3: 550-2.
9. Sikorska-Wisniewska G, Leberek A, Gebka MG. Mesenteric lymphadenopathy - a valid health problem in children. Med Wieku Rozwoj 2006; 10: 453-62.

10. Lee HJ. Plain abdominal radiography in infants and children. Korean J Pediatr Gastroenterol Nutr 2011; 14: 130-6.

11. Zviedre A, Engelis A, Tretjakovs P, Jurka A, Zile I, Petersons $A$. Role of serum cytokines in acute appendicitis and acute mesenteric lymphadenitis among children. Medicina 2016; 52: 291-7.

12. Rao PM, Rhea JT, Novelline RA. CT diagnosis of mesenteric adenitis. Radiology 1997; 202: 145-9.

13. Yazici M, Ozkisacik S, Oztan MO, Gürsoy H. Neutrophil/ lymphocyte ratio in the diagnosis of childhood appendicitis. Turk J Pediatr 2010; 52: 400-3.

14. Naess A, Nilssen SS, Mo R, Eide GE, Sjursen H. Role of neutrophil to lymphocyte and monocyte to lymphocyte ratios in the diagnosis of bacterial infection in patients with fever. Infection 2017; 45: 299-307.

15. Rothrock SG, Skeoch G, Rush JJ, et al. Clinical features of misdiagnosed appendicitis in children. Ann Emerg Med 1991; 20: 45-50.

16. Alvarado A. A practical score for the early diagnosis of acute appendicitis. Ann Emerg Med 1986; 15: 557-64.

17. Samuel M. Pediatric appendicitis score. J Pediatr Surg 2002; 37: 877-81.

18. Bhatt M, Joseph L, Ducharme FM, et al. Prospective validation of the pediatric appendicitis score in a Canadian pediatric emergency department. Acad Emerg Med 2009; 16: 591-6.

19. Goldman RD, Carter S, Stephens D, et al. Prospective validation of the Pediatric Appendicitis Score. J Pediatr 2008; 153: 278-82.

20. Bachur R. Evaluating appendicitis scoring systems using a prospective pediatric cohort. Ann Emerg Med 2007; 49: 778-84.

21. Lee HJ. Plain abdominal radiography in infants and children. Korean J Pediatr Gastroenterol Nutr 2011; 14: 130-6.

22. Karmazyn B, Werner EA, Rejail B, Applegate KE. Mesenteric lymph nodes in children: what is normal? Pediatr Radiol 2005; 35: 774-7.

23. Rothrock SG, Pagane J. Acute appendicitis in children: emergency department diagnosis and management. Ann Emerg Med 2000; 36: 39-51.

24. Wędrychowicz A, Tomasik P, Zając A, Fyderek K. Prognostic value of assessment of stool and serum IL-1beta, IL1ra and IL- 6 concentrations in children with active and inactive ulcerative colitis. Arch Med Sci 2018; 14: 107-14.

25. Akbulut UE, Emeksiz HC, Kocak FG, Livaoglu A. Diagnostic yield of esophagogastroduodenoscopy in children with chronic abdominal pain. Arch Med Sci 2018; 14: 74-80.

26. Adamczuk D, Roszkowska-Blaim M. Long-term outcomes in children with chronic kidney disease stage 5 over the last 40 years. Arch Med Sci 2017; 13: 635-44.

27. Ashindoitiang JA, Atoyebi AO, Arogundade RA. The value of plain abdominal radiographs in management of abdominal emergencies in Luth. Nig Q J Hosp Med 2008; 18: $170-4$ 